\title{
Differential priming effect for subliminal fear and disgust facial expressions
}

\author{
Su Young Lee • Jee In Kang • Eun Lee • \\ Kee Namkoong • Suk Kyoon An
}

Published online: 10 November 2010

(C) Psychonomic Society, Inc. 2010

\begin{abstract}
Compared to neutral or happy stimuli, subliminal fear stimuli are known to be well processed through the automatic pathway. We tried to examine whether fear stimuli could be processed more strongly than other negative emotional stimuli using a modified subliminal affective priming paradigm. Twenty-six healthy subjects participated in two separated sessions. Fear, disgust and neutral facial expressions were adopted as primes, and 50\% happy facial stimuli were adopted as a target to let only stronger negative primes reveal a priming effect. Participants were asked to appraise the affect of target faces in the affect appraisal session and to appraise the genuineness of target faces in the genuineness appraisal session. The genuineness instruction was developed to help participants be sensitive to potential threats. In the affect appraisal, participants judged $50 \%$ happy target faces significantly more 'unpleasant' when they were primed by fear faces than primed by $50 \%$ happy control faces. In the genuineness appraisal, participants judged targets significantly more 'not genuine' when they were primed by fear and disgust faces than primed by controls. These findings suggest that there
\end{abstract}

\author{
S. Y. Lee $\cdot$ E. Lee $\cdot$ K. Namkoong $(\bowtie) \cdot$ S. K. An \\ Department of Psychiatry, Severance Hospital, \\ Yonsei University College of Medicine, \\ 250 Seongsanno, Seodaemun-gu, \\ Seoul 120-752, Republic of Korea \\ e-mail: keen@yuhs.ac \\ S. Y. Lee $\cdot$ J. I. Kang $\cdot$ E. Lee $\cdot$ K. Namkoong $\cdot$ S. K. An \\ Institute of Behavioral Science in Medicine, \\ Yonsei University College of Medicine, \\ Seoul, Korea \\ J. I. Kang \\ Department of Psychiatry, Ilsan Hospital, \\ National Health Insurance Corporation, \\ Gyeonggi-do, Korea
}

may be differential priming effects between subliminal fear and disgust expressions, which could be modulated by a sensitive context of potential threat.

Keywords Subliminal priming effect · Fear - Disgust . Affect appraisal - Genuineness appraisal

\section{Introduction}

Fear is one of human's basic emotions. It is categorized as a negative emotion that makes us tense and nervous (Ekman $\&$ Friesen, 1969). From a functional perspective, however, fear is essential for our survival because it allows us to react against potential threats. To perceive and respond faster, the brain has evolved an automatic pathway that allows subtle, subliminal, and unconscious fear stimuli to be processed without cognitive participation. Many studies have argued that subliminal fear stimuli could evoke stronger responses and brain activities than neutral or happy stimuli. Fearful pictures, not neutral pictures, increased skin conductance responses (SCRs) even though participants could not recognize the masked stimuli (Najström \& Jansson, 2006). Undetected fear facial expressions elicited greater eventrelated potential (ERP) than neutral stimuli (Eimer \& Kiss, 2008; Gordon, Shevrin, Rathjen, Williams, \& Liddell, 2004) as supraliminal fear faces did (An et al., 2003). Imaging studies demonstrated that subliminal fear stimuli could activate subcortical structures such as the amygdala by passing higher cortical structures (Liddell et al., 2005; Williams et al., 2006). These findings implied that the fear emotion activated subliminal processing as an alarm for the threat. However, in previous studies, subliminal fear stimuli were compared with neutral or happy stimuli, not with other negative emotions. There were findings suggesting 
that global emotional valence could be processed on the subliminal level (Murphy \& Zajonc, 1993; Ruys \& Stapel, 2008a); therefore it has been unclear whether the stronger subliminal processing of fear was derived from a global negative valence or a threatening nature of the emotion. In this study, we tried to compare subliminal fear processing with other negative emotions using a representative experiment assessing subliminal processing - a priming paradigm.

Subliminal affective priming means that a brief emotional stimulus that is below the visual detection threshold could generate a significant shift in the participants' judgment for followed supraliminal ambiguous targets. Murphy and Zajonc (1993) developed this paradigm, and in their original study, their participants judged a Chinese character (a target), which was a novel and ambiguous stimuli for Caucasian participants, as a positive one when subliminal (4 ms) happy facial primes were previously given (positive judgment shift), and judged as negative when negative (sad, anger, or fear) facial primes were given (negative judgment shift) (Murphy \& Zajonc, 1993). The authors explained that subliminal input of emotional stimuli could elicit an early emotional reaction without conscious awareness, and this reaction automatically spread to another following conscious judgment. There have been interesting studies that applied subliminal affective priming to clinical samples. People with schizophrenia showed a stronger subliminal priming effect for negative emotions than healthy controls (Höschel \& Irle, 2001), and people with pervasive developmental disorder did not show a priming effect for fear, whereas healthy controls did (Fein, Wolf, \& Kamio, 2006). These studies implicated that subliminal affective processing would be related to psychopathologies such as sensitivity to negative emotion in schizophrenia or deficits in pervasive developmental disorder. However, no studies have demonstrated differential priming effects within negative emotional stimuli. It could provide further impressions for psychopathologies to understand whether this priming effect is limited to subliminal fear or shared with other negative emotions.

In this study, we investigated whether the subliminal fear expression could make stronger priming effects than other negative expressions or not. We tried to reveal the difference in priming effects within the emotions with negative valence of a type more clearly. First, we selected disgust as a contrast of the fear stimuli according to the previous emotion studies (Phillips et al., 1999; Williams et al., 2007). Researchers preferred disgust to sadness or anger because a specific neural correlate for disgust (i.e., insula) was clearly distinguished from that of fear (i.e., amygdala) (Phillips et al., 1997, 2004). Second, we adopted a slightly positive facial expression as a target (50\% happy face from morphing procedure) (Calder, Young, Rowland, \& Perrett, 1997). If a subsequent conscious target has positive valence, an early negative emotional reaction evoked by a negative subliminal prime would be diluted (Murphy \& Zajonc, 1993). So, we expected that only a stronger negative emotional prime could exclusively have a subliminal priming effect on a positive target. Third, we developed an additional instruction to make participants more sensitive to subtle fear stimuli - $a$ genuineness appraisal (i.e., "whether genuinely expressing a happy face to participants or not"). The priming effect was known to be influenced by experimental instructions. Participants' increased attention or expectation for certain contexts evoked by an instruction could strengthen the priming effects (Winkielman, Zajonc, \& Schwarz, 1997). For example, the priming effect was strengthened when the participants were informed that something would pass before the target for a short duration. We expected that the instruction that made them suspect the genuineness of the affect could evoke sensitivity to a threatening emotion - fear. We expected that while the affect appraisal would be casual in common social contexts, a genuineness appraisal would let participants be more sensitive to subliminal expression of fear, which could more robustly reveal the priming effect for subliminal expression of fear.

We hypothesized that the subliminal fear prime would show a stronger priming effect than other negative (disgust) and neutral primes. We also expected that this priming effect for fear would be more strongly presented in the genuineness session.

\section{Method}

\section{Participants}

Twenty-six participants were recruited by an Internet advertisement. They ranged in age from 19 to 49 years old (mean age $=31.6$ years, $\mathrm{SD}=7.2$ ). Thirteen of them were male, and the rest were female. All were right-handed and given more than 12 years of education. Psychiatric illness was ruled out by a psychiatrist (S.Y. Lee) using the Structured Clinical Interview for DSM-IV (SCID) (First, Spitzer, Gibbon, \& Williams 1996). Participants with Axis I or II disorders, neurological disorders, past histories of head injury, and relevant visual defects were excluded by an interview. This investigation was carried out in accordance with the American Psychological Association standards for ethical treatment of participants. This study was reviewed and approved by the Institutional Review Board of Yonsei University Health System. All participants signed a written informed consent.

Materials and apparatus

In this study, standardized Ekman photographs (Ekman, 1972) of facial expressions were used. To exclude a 
possible confounding effect of preference, we performed a preference assessment in eight healthy participants who were not part of the study population. We selected one female face (MO) that recorded the highest score as a target, and selected two male (JJ and PE) and two female ( $\mathrm{PF}$ and $\mathrm{SW}$ ) faces recorded as the next highest scores as primes. Hair, ears, and neck parts of each photograph were deleted to exclude an effect of other physical components except a facial expression. All photographs were monochrome against a black backdrop, and the size and brightness were equally adjusted. We used a gray scale vertical stripe pattern mask with different two tones (Fig. 1). The pictures were $12.4 \mathrm{~cm} \times 15.6 \mathrm{~cm}$ in size, and the distance between the monitor and participants was about $60 \mathrm{~cm}$. Stimuli were presented via a monitor with a refresh rate of $100 \mathrm{~Hz}$ [Magic SyncMaster CPG21s(T), Samsung, South Korea], and the priming experiment was realized by the software package Inquisit 2.0 [Inquisit 2.0.61004.3 (Computer software) 2006, Seattle, WA: Millisecond Software LLC.].

Since the target photograph had 50\% happy facial expression derived from a morphing procedure (Calder et al., 1997), we expected that only stronger negative primes such as fear stimuli would exclusively reveal subliminal priming effects. As experimental primes, we used $100 \%$ fear expressions, $100 \%$ disgust expressions, and neutral expressions of the selected two females (PF and SW) and two males (JJ and PE), and 50\% happy expressions of them as control primes.

Procedure

We arranged the sequence of stimuli to be in the order of a prime $(10 \mathrm{~ms})$, pattern mask $(10 \mathrm{~ms})$, and then a target ( $80 \mathrm{~ms})$ to make subliminal input of primes following the results of a previous study (Williams et al., 2004) which revealed the stimulus-onset asynchrony (SOA) of $10 \mathrm{~ms}$ as an unconscious detection threshold (Fig. 1). This sequence was repeated four times as a four-time repetition increased the probability of judgment shift and produced more homogeneous responses in a previous study (Höschel \& Irle, 2001; Pause, Ohrt, Prehn, \& Ferstl, 2004). Using this backward masking procedure, participants could consciously recognize only $50 \%$ happy target faces. To ensure that participants paid attention to the monitor during the brief subliminal exposure, a fixation cross was projected for $500 \mathrm{~ms}$ at the center of the monitor prior to the prime, signaling the start of each trial. The total duration time of each trial was $900 \mathrm{~ms}$ and the inter-trial interval was $4 \mathrm{~s}$.

The experiment consisted of three blocks of experimental primes (fear, disgust, or neutral) and 50\% happy control primes. The fear block consisted of fear and 50\% happy primes; the disgust block and neutral blocks were comprised in the same manner. Each block consisted of 16
Fig. 1 Illustration of each item of fear, disgust, and neutral block. The mask and the $50 \%$ happy target face were the same in each block

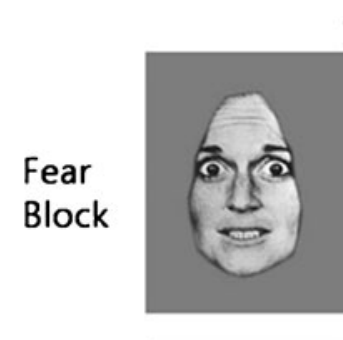

$10 \mathrm{~ms}$

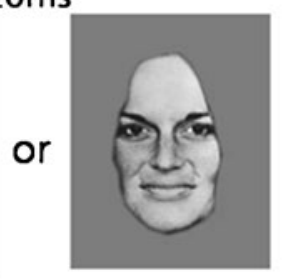

$10 \mathrm{~ms}$
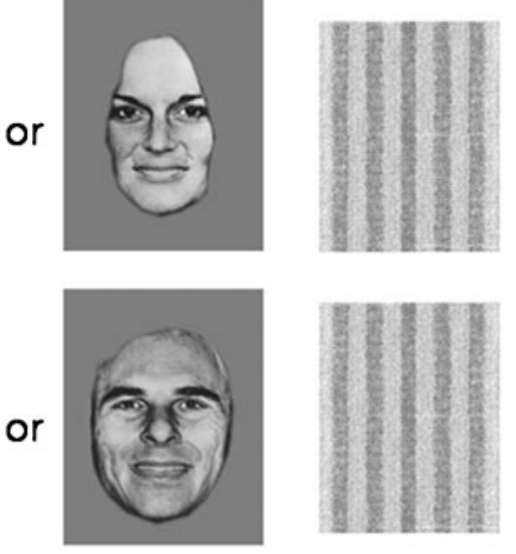

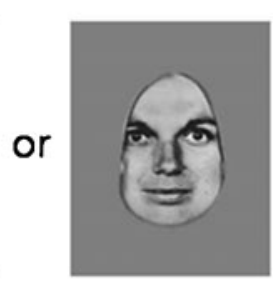

Experimental

prime
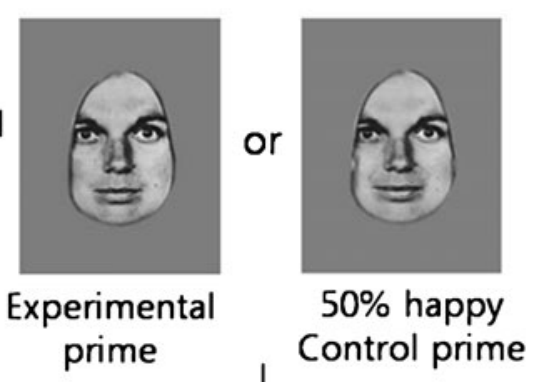

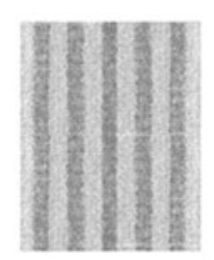

Mask
$80 \mathrm{~ms}$
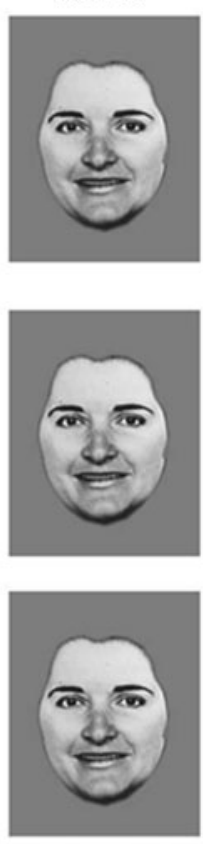

$50 \%$ happy

Target 
trials, and the experimental and the control primes were equally presented. The order of the block presentation was counterbalanced across the participants (Fig. 1).

Participants performed two separated sessions consisting of the same procedures but different appraisal instructions an affect appraisal for being a casual context and a genuineness appraisal for being a sensitive context to a potential threat. The question given in the affect appraisal session was "Does this person seem pleasant or unpleasant?" Because the target face expressed a slightly positive $(50 \%$ happy) emotion, participants were told that the woman in the photograph had been directed to wear a little smile, but her inner emotion could be either pleasant or unpleasant. The question for the genuineness appraisal session was "This person says 'I like you.' Does this person seem to really like you or not?" Participants were told that the woman was photographed when saying "I like you," and she may or may not mean it. Both instructions suggested a possibility of a concealed truth, but one was about the other's own feeling, another was about the other's feeling toward participant. We expected that the genuineness appraisal, which gives a color of a potential threat to the context, could make participants more attentive to the experiment and sensitive to subtle emotions, which in effect would strengthen the priming effects. They responded using "p" and "q" buttons on the keyboard, which were used as buttons for "pleasant" and "unpleasant" during the affect appraisal session and as buttons for "genuine" and "not genuine" during the genuineness session, respectively. The locations of buttons were counterbalanced across the participants. Since participants' sensitivity to fear evoked by the genuineness appraisal might influence the affect appraisal session, we did not counterbalance the order of sessions.

After the sessions, all participants reported whether they saw any faces other than a target face. Then, they were given a forced-choice test of awareness to verify the absence of conscious prime recognition. In this test, participants were informed that they would be given other faces (primes) very briefly before the target face. The faces were presented in the order of a prime $(10 \mathrm{~ms})$, mask $(10 \mathrm{~ms})$, and then the target $(80 \mathrm{~ms})$ for four times, in the same manner for the priming task. The sequence was followed by two faces for $2,000 \mathrm{~ms}$ - an actual prime and an alternative face that matched in gender and valence to an actual prime. Participants were then asked to pick the prime between the two faces. A total of 20 trials were carried out. If participants could not consciously recognize the prime, they should not do better than a chance level responding.

Finally, a valence recognition task (a facial expression verification task) was carried out. All primes and target faces used in the current study were presented to participants as targets when they were asked to value each target as positive, negative, or neutral using the "i", "o," and "p" buttons on the keyboard. A sequence of vertical striped mask (10 ms) horizontal striped mask $(10 \mathrm{~ms})$ - target $(80 \mathrm{~ms})$ was presented for four times to make the same condition as the actual experiment. This verification task was conducted to verify the premise that participants appropriately perceived emotional valences of the faces.

Psychological measures

The Beck Depression Inventory (BDI) (Beck, Ward, Mendelson, Mock, \& Erbaugh, 1961) and state anxiety of Spielberger's State and Trait Anxiety Inventory (STAI) (Lee \& Won, 1995; Spielberger, Gorsuch, \& Lushene 1970) were used for all participants in order to evaluate the baseline mood and anxiety state that can influence their affect and genuineness appraisals. The paranoia scale (Fenigstein \& Vanable, 1992) and Ambiguous Intentions Hostility Questionnaire (AIHQ) (Chang et al., 2009; Combs, Penn, Wicher, \& Waldheter, 2007) were performed to evaluate their sensitivity to social threat, and the disgust scale (Haidt, McCauley, \& Rozin, 1994) was used to assess their sensitivity to disgust.

Data reduction and statistical analysis

We assessed the priming effect for each block using discrimination sensitivity $\left(d^{\prime}\right)$ in the signal detection theory (Macmillan \& Creelman, 2005) in accordance with a previous priming study (Greenwald, Draine, \& Abrams, 1996). The $d$ ' is a value reflecting participants' discrimination accuracy between two stimuli controlling the response bias. A value of 0 for $d^{\prime}$ corresponds to the correct percentage of $50 \%$, which means that participants responded equally, and were not able to discriminate the two stimuli at all. The $d^{\prime}$ values between 0.5 and 2.5 roughly correspond to correct percentages between $60 \%$ and $90 \%$. Because the priming is a subtle and implicit process, participants might often be unsure of their judgment. When participants could not distinguish the two stimuli well, they tended to rely on their internal response criterion rather than objective standards. For example, if someone had an extreme negative response bias and responded negatively to all stimuli, his negative response rate of that block would be $100 \%$, whereas the discrimination sensitivity would be 0 . Furthermore, the $d$ ' value was an appropriate measure for this study because it is invariant when the response bias was affected by the manipulation of instruction. That is, when a different priming effect between the two appraisal sessions was revealed, the $d$ ' value could be helpful in verifying whether this difference was due to a change in the response criterion or a change in the priming effect itself.

The stimulus-response matrix for the current task is demonstrated in Table 1. The $d^{\prime}$ is defined in terms of the $z$ 
Table 1 Stimulus-response matrix of affect/genuineness appraisal tasks

\begin{tabular}{lll}
\hline Stimulus class & \multicolumn{2}{l}{ Response for target } \\
\cline { 2 - 3 } & Unpleasant/not genuine & Pleasant/genuine \\
\hline Experimental prime (fear, disgust, or neutral) & Hits & Misses \\
Control prime (50\% happy) & False alarms & Correct rejections \\
\hline
\end{tabular}

score (i.e., standard deviation units), which is converted from a hit and a false alarm rate by the $z$ transformation as follows.

$d^{\prime}=z(H)-z(F) \quad(H ;$ hit rate, $F ;$ false alarm rate $)$

Therefore, if more participants appraised the affect or genuineness of a target face as being 'unpleasant' or 'not genuine' with experimental primes (fear, disgust, and neutral) rather than with $50 \%$ happy control primes in each block, then more discrimination sensitivity $\left(d^{\prime}\right)$ of that block would have a greater positive value. The discrimination sensitivity $\left(d^{\prime}\right)$ over 0.5 was considered as a meaningful priming effect, which is better than a chance level (i.e., $d^{\prime}=0$ ) in the discrimination analysis (Macmillan $\&$ Creelman, 2005). To make sure that the $d$ ' values of the affect and genuineness appraisal tasks were significantly greater than zero, we conducted one-sample t-tests (Table 2).

The $d^{\prime}$ values were screened for outliers using Tukey's hinges, and, when possible, outliers were included after a Winsorization procedure (Hoaglin, Mosteller, \& Tukey, 1983). Following data cleaning, all $d^{\prime}$ values had acceptable skewness statistics $(<1.0)$. To compare the discrimination sensitivity of the three blocks (neutral, disgust, and fear), repeated-measures analysis of variance (ANOVAs) and paired post-hoc comparisons with a Bonferroni correction were performed for each session. To assess the correlations between discrimination sensitivity $\left(d^{\prime}\right)$ and psychological measures, Pearson procedures were performed. Otherwise, the appropriate paired t-test was performed.

\section{Results}

Affect appraisal session

The mean discrimination sensitivity $\left(d^{\prime}\right)$ was $0.23(\mathrm{SD}=$ $0.57)$ for the neutral block, $0.15(\mathrm{SD}=0.44)$ for the disgust block, and $1.30(\mathrm{SD}=0.83)$ for the fear block. The mean $d^{\prime}$ of the fear block 1.30 suggested that there was a significant discrimination - a priming effect (Table 2). Repeated measure ANOVAs showed a significant block difference $(F(2,24)=$ 30.81 , MSE $=0.35, p<0.001$ ), and post-hoc analysis showed that this difference was derived from significantly higher discrimination sensitivity $\left(d^{\prime}\right)$ in the fear block than the disgust and the neutral block (fear $>$ disgust, $p<0.001$; fear $>$ neutral, $p<0.001$ ) (Fig. 2).

Genuineness appraisal session

The mean discrimination sensitivity $\left(d^{\prime}\right)$ was 0.07 ( $\mathrm{SD}=$ $0.54)$ for the neutral block, $0.59(\mathrm{SD}=0.79)$ for the disgust block, and $1.14(\mathrm{SD}=0.92)$ for the fear block. The mean $d^{\prime}$ of the fear block 1.14 and that of disgust block 0.59 suggested that fear and disgust primes produced significant priming effects (Table 2). Repeated measure ANOVAs showed a significant block difference $(\mathrm{F}(2,24)=12.70$, MSE $=0.58, p<0.001)$, and post-hoc analysis showed that the fear block and the disgust block showed significantly higher discrimination sensitivity $\left(d^{\prime}\right)$ than the neutral block (fear $>$ neutral, $p<0.001$; disgust $>$ neutral, $p=0.044$ ). There was a trend level difference between the disgust block and the fear block ( $p=0.066)$ (Fig. 2).

Table 2 The proportion of unpleasant/not genuine responses for the target and discrimination sensitivities $\left(d^{\prime}\right)$ in each block of the affect and the genuineness appraisal session

\begin{tabular}{|c|c|c|c|c|c|c|}
\hline \multirow[t]{2}{*}{ Session } & \multirow[t]{2}{*}{ Block } & \multirow{2}{*}{$\frac{\text { Experimental prime (\%) }}{\text { Mean (SD) }}$} & \multirow{2}{*}{$\frac{\text { Control prime (\%) }}{\text { Mean (SD) }}$} & \multicolumn{3}{|c|}{ Discrimination sensitivities $\left(d^{\prime}\right)$} \\
\hline & & & & Mean (SD) & $t, d f^{*}$ & $P^{*}$ \\
\hline \multirow[t]{3}{*}{ Affect } & Neutral & $31.3(31.5)$ & $24.0(28.9)$ & $0.23(0.57)$ & $1.70,25$ & 0.101 \\
\hline & Disgust & $34.6(34.7)$ & $29.8(35.5)$ & $0.15(0.44)$ & $1.18,25$ & 0.250 \\
\hline & Fear & $62.5(22.1)$ & $19.2(27.9)$ & $1.30(0.83)$ & $7.96,25$ & $<.001$ \\
\hline \multirow[t]{3}{*}{ Genuineness } & Neutral & $30.8(30.0)$ & $28.6(25.5)$ & $0.07(0.54)$ & $0.69,25$ & 0.500 \\
\hline & Disgust & $43.3(36.6)$ & $23.6(26.1)$ & $0.59(0.79)$ & $3.74,25$ & 0.001 \\
\hline & Fear & $65.4(21.3)$ & $27.9(26.8)$ & $1.14(0.92)$ & $5.96,25$ & $<.001$ \\
\hline
\end{tabular}

*One-sample t-test to examine whether the $d$ ' values of the affect and genuineness appraisal tasks were significantly greater than 'zero' 


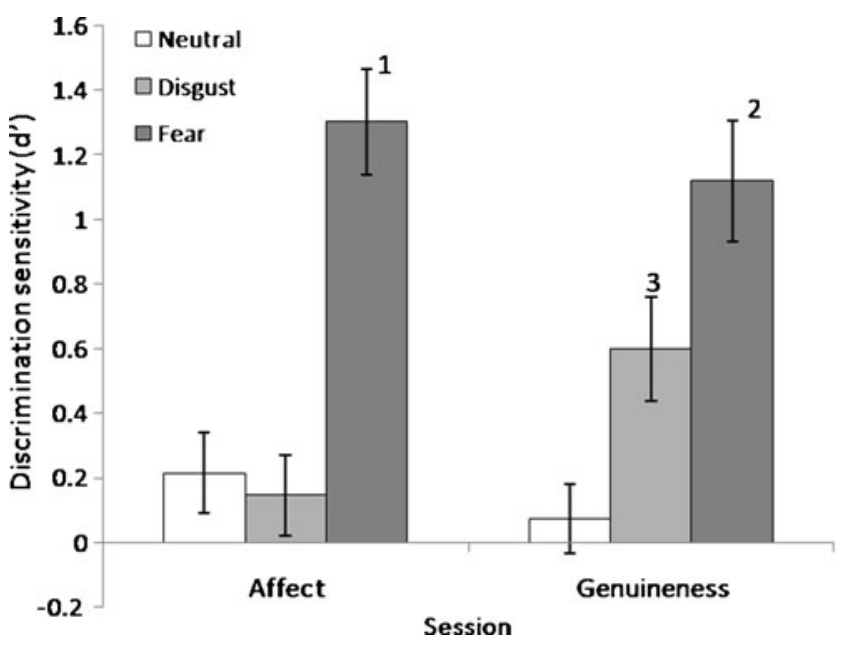

Fig. 2 Mean of the discrimination sensitivity $\left(d^{\prime}\right)$ with standard error in the affect appraisal session and the genuineness appraisal session. In the affect appraisal session, the mean of discrimination sensitivity $\left(d^{\prime}\right)$ in the fear block ${ }^{1}$ was significantly higher than in the disgust and the neutral block (fear $>$ neutral: $p<0.001$, fear $>$ disgust: $p<0.001$ ). In the genuineness appraisal session, the mean of discrimination sensitivity $\left(d^{\prime}\right)$ in the fear block ${ }^{2}$ and the disgust block ${ }^{3}$ were significantly higher than that in the neutral block (fear $>$ neutral: $p<$ 0.001 , disgust $>$ neutral: $p=0.044$ )

There was a significant difference between the discrimination sensitivity of the disgust block between the affect and genuineness appraisal session (paired- $\mathrm{t}=-3.08, p=0.005$ ), but there was no significant difference for the fear and neutral blocks between the two sessions (for fear block, paired $\mathrm{t}=$ $0.94, p=0.357$; for neutral block, paired $\mathrm{t}=0.85, p=0.404$ ).

Forced-choice test of awareness and valence recognition task

According to the forced-choice test of awareness, the mean correct response was $60.2 \%(\mathrm{SD}=12.0)$. To test whether this response rate was significantly better than a chance level, the t-test from the discrimination analysis was performed. As a result, the mean correct response of $60.2 \%$ was not significantly better than a chance level $(\mathrm{t}=$ $0.901, \mathrm{df}=18, p=0.190)$. Two participants recorded $80 \%$ correct response, but they said that they had not recognized any other faces besides a target face during the task. However, to ensure that our task was subliminal, we additionally performed median-split analysis. As a result, there was no significant difference in discrimination sensitivity $\left(d^{\prime}\right)$ for each emotion between the above median group and the below median group in both sessions [in affect session - neutral $(p=0.992)$; disgust $(p=0.231)$; fear $(p=0.474)$, in genuineness session - neutral $(p=$ $0.943)$; disgust $(p=0.250)$; fear $(p=0.610)]$.

As a result of the valence recognition task, participants mostly recognized the $50 \%$ happy faces as positive (mean $=$
$81.3 \%, \mathrm{SD}=16.9)$, neutral face as neutral $($ mean $=86.1 \%$, $\mathrm{SD}=16.8)$, disgust $($ mean $=77.8 \%, \mathrm{SD}=34.6)$, and fear face $($ mean $=94.4, \mathrm{SD}=16.1)$ as negative.

Correlations with psychological measures

There was no significant correlation between psychological measures (BDI, state anxiety from STAI, paranoia scale, AIHQ, and disgust scale) and discrimination sensitivity $\left(d^{\prime}\right)$ of the fear block in the affect appraisal session and the fear and disgust block in the genuineness session. The mean score of BDI and state anxiety of this sample were $7.69 \pm$ 6.92 and $40.73 \pm 8.30$, respectively.

\section{Discussion}

In this study, we examined if a facial expression of fear could make a stronger subliminal priming effect than other negative facial expressions. In the affect appraisal session, a fear expression revealed a significantly stronger subliminal priming effect than disgust and neutral expressions. As far as we know, this was the first report that showed the stronger priming effect for fear emotional stimuli distinguished from other negative stimuli. This finding is consistent with previous evidence about activation of an automatic pathway by subliminal fear stimuli, such as eliciting autonomic response and brain activities (Gordon et al., 2004; Liddell et al., 2005; Najström \& Jansson, 2006). Since one important role of the automatic pathway is to rapidly detect potential danger, the threatening nature of the fear expression (Bradley, Garner, \& Mogg, 2007; Detweiler-Bedell \& Becker, 2009) seems to contribute to the differential priming effect for this emotion. In addition, the current study showed that subliminal fear expression could make a priming effect on a slightly positive target (50\% happy face). This finding suggests that fear expression may be a strong enough alarm to shift a person's judgment on a slightly positive target. Taken together, the findings of the affect appraisal session suggest that fear seems to be stronger and an automatic-proper emotion compared to other non-threatening negative emotions (disgust) in the casual social context, and this priming paradigm using a slightly positive target (50\% happy face) could be an effective tool to reveal a stronger priming effect for fear.

In a genuineness appraisal session, both subliminal fear and disgust emotions revealed significant priming effects. The fear emotion repeatedly made a significant priming effect on a positive target. We had the genuineness appraisal session to make participants be more directly placed toward a potential threat (social rejection) and be more sensitive to subliminal fear stimuli than those in the 
affect appraisal session. We expected that this instruction would reveal a stronger priming effect for fear primes, but the priming effects for fear in the affect and genuineness session did not show a significant difference. To explain this result, we could consider some possibilities. First, participants' early emotional reactions induced by fear prime in both sessions might not be significantly different in terms of the intensity for the priming effect. Another possibility is a ceiling effect. The mean discrimination sensitivity of the fear block (1.30) in the affect appraisal session roughly corresponded to a correct percentage of $74 \%$ and that of the neutral block $(0.21)$ corresponded to $54 \%$. If we substitute this value for a judgment shift index in a previous priming experiment (Höschel \& Irle, 2001) using a very similar subliminal priming paradigm, the negative shift by fear prime in our study was about $20 \%$, and the negative judgment shift by various negative primes (fear, disgust, sad) in the previous experiment was 13\%. We cannot directly compare the two studies, but a $20 \%$ shift might be too high. That is, the priming effect for fear might already have sufficiently appeared in the affect appraisal session. Third, our instruction for the genuineness session might have failed to differentiate a priming effect. However, this instruction seems to have an effect because the priming effect for disgust changed across the two sessions. Altogether the fear emotion repeatedly made a stronger priming effect than disgust in the trend level and neutral in the significant level. This finding means that fear might also be stronger than other negative emotional expressions in terms of sensitivity to a potential threat.

The appearance of a priming effect for disgust in the genuineness session, which had not existed in the affect appraisal session, was an unexpected finding, but it provides us interesting impressions. Disgust has been known as a non-threatening negative emotion related to withdrawal from distaste or contamination. However, withdrawal from "an unfavorable person" could be a "rejecting" emotion in a social dimension (Rozin, Haidt, $\&$ McCauley, 2000; Taylor, 2007). Seeing someone who is disgusting for something may not be a threat to an observer in a casual context, but seeing someone who expresses disgust to an observer him- or herself could be a threat or fear in a social context (Peer, Rothmann, Penrod, Penn, \& Spaulding, 2004). We speculated that the instruction in the genuineness session - "This person says 'I like you'. Does this person seem to really like you or not?" - might make an early emotional reaction for disgust act as a social threat, which in turn activates the automatic system. Another possible explanation is that the newly observed priming effect of disgust in the genuineness session might be attributed to the globally strengthened sensitivity to all negative emotions that was evoked by the genuineness instruction. Global, valence-based negative mood response could be evoked by subliminal priming (duration of prime $=$ $40 \mathrm{~ms}$ ), whereas the emotion-specific response (disgust, fear, anger - specific response) could not be subliminally primed (Ruys \& Stapel, 2008a, 2008b). However, we observed a stronger fear priming effect compared to the priming effect for disgust in the affect appraisal session. Considering Ruys' study, we speculated that it might be due to some valence-based nature of fear rather than a categorical difference between fear and disgust. The difference in instructions between our study and Ruys' study might provide some clues for this issue. The instruction in Ruys' study was to evaluate the participant's (receiver's) own feeling, whereas our instruction was to evaluate other's (sender's) affect. It might be enough for one to feel global negative emotion when he sees other's fearful face, but it would be more critical to catch the potential threats. Therefore, a common reason for the differential priming effect for fear in the affect appraisal and the priming effect for disgust and fear in the genuineness appraisal might be the negative mood reaction, which serves as an alarm to potential social threats. Further studies applying this priming paradigm to sadness might be helpful to clarify this point.

Psychological measures showed no correlations with priming effects in both sessions. Depression and anxiety did not seem to influence priming effects. The paranoia scale and the AIHQ, which were performed to measure sensitivity of social threat, did not show any correlation with the priming effects. These scales might not reflect the implicit sensitivities for threat or might have limitations to measure discriminative sensitivity among healthy normal subjects. The disgust scale also did not show a correlation with a priming effect for disgust. This might be because the disgust scale measures the sensitivity to core disgust or contamination, so it might not be suitable to measure the sensitivity of disgust in a social dimension, which seemed to make a priming effect in this study. However, the relatively small sample size might be a crucial factor for no correlations between psychological measures and priming effects. If some significant clinical correlations are found in a future study with a larger sample, the appearance of a priming effect in the genuineness session may be further explained.

We have to consider the findings of the present study in light of several limitations. The mean correct response of the forced-choice test of awareness seems to be somewhat high $(60.2 \%)$. Even though all participants answered that they did not see any face other than the target face, we performed a median-split analysis to examine whether the results of the forced-choice test influenced the priming effect. As a result, there was no significant difference in discrimination sensitivity $\left(d^{\prime}\right)$ between the above median group and the below median group in both sessions. In 
addition, if participants could consciously recognize the primes, the priming effect would be produced not only by the fear prime but also by the disgust prime in the affect appraisal session. Therefore, this limitation might not fully negate our results. Second, the mean recognition rate for happy face $(81.3 \%)$ was a bit lower, but it may be because we used $50 \%$ happy faces, not $100 \%$ happy faces, which have usually showed near $100 \%$ recognition rate. A lower mean recognition rate for disgust faces (77.8\%) rather than for fear faces (94.4\%) could also be a limitation. We cannot rule out the possibility that the differential priming effect in the affect appraisal was due to the differential recognition rates. We additionally analyzed the priming effect of participants whose recognition rate for disgust was $100 \%$ $(N=18)$, and the overall results were consistent with the original results ( $d$ ' for disgust in the affect appraisal session $=$ 0.13 ; $d$ ' for genuineness appraisal session $=0.53$ ). Thus, the relatively lower recognition rate for disgust stimuli might not be enough to change our main findings that the differential priming effects between subliminal fear and disgust expression could be modulated by a sensitive context of a potential threat.

In conclusion, our results demonstrated a stronger subliminal priming effect for fear compared with other negative expression (disgust). We also showed that subliminal fear could make a priming effect even on a slightly positive target (50\% happy face). Furthermore, we showed that the manipulation of instructions could change the priming effect for the same affect (disgust). This finding suggested that participants' previous attention or the context related to the prime could influence the following automatic processing of affects. In the current study, the sensitive context for a potential threat in the genuineness session seems to influence the priming effect. Applying this paradigm to the patients group who are sensitive to fear stimuli or social rejection such as social phobia or schizophrenia, we might find further implications about the priming effect and insights into the connection between psychopathologies and automatic processing of emotions.

Acknowledgements This study was supported by a grant from the Health and Medical Technology R\&D Program of the Ministry for Health, Welfare and Family Affairs of the Republic of Korea (A080713).

\section{References}

An, S. K., Lee, S. J., Lee, C. H., Cho, H. S., Lee, P. G., Lee, C. I., et al. (2003). Reduced P3 amplitudes by negative facial emotional photographs in schizophrenia. Schizophrenia Research, 64(2-3), $125-135$.

Beck, A. T., Ward, C. H., Mendelson, M., Mock, J., \& Erbaugh, J. (1961). An inventory for measuring depression. Archives of General Psychiatry, 4, 561-571.
Bradley, B. P., Garner, M., \& Mogg, K. (2007). Anxiety and orienting of gaze to angry and fearful faces. Biological Psychology, 76(3), $163-169$.

Calder, A. J., Young, A. W., Rowland, D., \& Perrett, D. I. (1997). Computer-enhanced emotion in facial expressions. Proceedings of the Royal Society B: Biological Sciences, 264(1383), 919-925. doi:10.1098/rspb.1997.0127

Chang, H. Y., Lee, S. K., Kim, K. R., Lee, S. Y., Park, J. Y., Kim, E. J., et al. (2009). Development of Korean version of the ambiguous intentions Hostility Questionnaire(K-AIHQ). Journal of Korean Neuropsychiatric Association, 48, 29-35.

Combs, D. R., Penn, D. L., Wicher, M., \& Waldheter, E. (2007). The Ambiguous Intentions Hostility Questionnaire (AIHQ): A new measure for evaluating hostile social-cognitive biases in paranoia. Cognitive Neuropsychiatry, 12(2), 128-143. doi:10.1080/ 13546800600787854

Detweiler-Bedell, B., \& Becker, M. W. (2009). Early detection and avoidance of threatening faces during passive viewing. The Quarterly Journal of Experimental Psychology, 62(7), $1257-1264$

Eimer, M., \& Kiss, M. (2008). ERPs reveal subliminal processing of fearful faces. Psychophysiology, 45(2), 318-326.

Ekman, P. (1972). Universals and cultural differences in facial expressions of emotions (Vol. 19). University of Nebraska Press.

Ekman, P., \& Friesen, W. V. (1969). The repertoire of nonverbal behavior: Categories, origins, usage, and coding. Semiotica, 1, 49-98.

Fein, D., Wolf, J., \& Kamio, Y. (2006). Automatic processing of emotional faces in high-functioning pervasive developmental disorders: An affective priming study. Journal of Autism and Developmental Disorders, 36(2), 155-167.

Fenigstein, A., \& Vanable, P. A. (1992). Paranoia and selfconsciousness. Journal of Personality and Social Psychology, 62(1), 129-138.

First, M. B., Spitzer, R. L., Gibbon, M., \& Williams, J. B. (Eds.). (1996). Structured Clinical Interview for DSM-IV Axis I Disorders: Patient Edition (SCID-I/P). Version 2: Biometrics Research, New York.

Gordon, E., Shevrin, H., Rathjen, J., Williams, L. M., \& Liddell, B. J. (2004). A temporal dissociation of subliminal versus supraliminal fear perception: An event-related potential study. Journal of Cognitive Neuroscience, 16(3), 479-486.

Greenwald, A. G., Draine, S. C., \& Abrams, R. L. (1996). Three cognitive markers of unconscious semantic activation. Science, 273(5282), 1699-1702.

Haidt, J., McCauley, C., \& Rozin, P. (1994). Individual differences in sensitivity to disgust: A scale sampling seven domains of disgust elicitors. Personality and Individual Differences, 16, 701-713.

Hoaglin, D., Mosteller, F., \& Tukey, J. (1983). Understanding Robust and Exploratory Data Analysis. New York: Wiley.

Höschel, K., \& Irle, E. (2001). Emotional priming of facial affect identification in schizophrenia. Schizophrenia Bulletin, 27(2), 317-327.

Lee, H. J., \& Won, H. (1995). A study of the reliability and the validity of the paranoia scale. Korean Journal of Clinical Psychology, 14, 83-94.

Liddell, B. J., Brown, K. J., Kemp, A. H., Barton, M. J., Das, P., Peduto, A., et al. (2005). A direct brainstem-amygdala-cortical 'alarm' system for subliminal signals of fear. Neuroimage, 24(1), 235-243.

Macmillan, N. A., \& Creelman, C. D. (2005). Detection theory, a user's guide (2nd ed.). Mahwah: Lawrence Erlbaum Associates, Inc.

Murphy, S. T., \& Zajonc, R. B. (1993). Affect, cognition, and awareness: Affective priming with optimal and suboptimal stimulus exposures. Journal of Personality and Social Psychology, 64(5), 723-739.

Najström, M., \& Jansson, B. (2006). Unconscious responses to threatening pictures: Interactive effect of trait anxiety and social 
desirability on skin conductance responses. Cognitive Behaviour Therapy, 35(1), 11-18.

Pause, B. M., Ohrt, A., Prehn, A., \& Ferstl, R. (2004). Positive emotional priming of facial affect perception in females is diminished by chemosensory anxiety signals. Chemical Senses, 29(9), 797-805. doi:10.1093/chemse/bjh245

Peer, J. E., Rothmann, T. L., Penrod, R. D., Penn, D. L., \& Spaulding, W. D. (2004). Social cognitive bias and neurocognitive deficit in paranoid symptoms: Evidence for an interaction effect and changes during treatment. Schizophrenia Research, 71(2-3), 463-471.

Phillips, M. L., Williams, L. M., Heining, M., Herba, C. M., Russell, T., Andrew, C., et al. (2004). Differential neural responses to overt and covert presentations of facial expressions of fear and disgust. Neuroimage, 21(4), 1484-1496.

Phillips, M. L., Williams, L., Senior, C., Bullmore, E. T., Brammer, M. J., Andrew, C., et al. (1999). A differential neural response to threatening and non-threatening negative facial expressions in paranoid and non-paranoid schizophrenics. Psychiatry Research, 92(1), 11-31.

Phillips, M. L., Young, A. W., Senior, C., Brammer, M., Andrew, C., Calder, A. J., et al. (1997). A specific neural substrate for perceiving facial expressions of disgust. Nature, 389(6650), 495498. doi:10.1038/39051

Rozin, P., Haidt, J., \& McCauley, C. (2000). Disgust. In M. L. J. Haviland (Ed.), Handbook of emotions (pp. 637-652). New York: Guilford Press.
Ruys, K. I., \& Stapel, D. A. (2008a). Emotion elicitor or emotion messenger? Subliminal priming reveals two faces of facial expressions. Psychological Science, 19(6), 593-600.

Ruys, K. I., \& Stapel, D. A. (2008b). How to heat up from the cold: Examining the preconditions for (unconscious) mood effects. Journal of Personality and Social Psychology, 94(5), 777-791.

Spielberger, G., Gorsuch, R. L., \& Lushene, R. E. (1970). Manual for the State-Trait anxiety inventory. Palo Alto: Consulting Psychologists Press.

Taylor, K. (2007). Disgust is a factor in extreme prejudice. The British Journal of Social Psychology, 46(3), 597-617.

Williams, L. M., Das, P., Liddell, B. J., Olivieri, G., Peduto, A. S., David, A. S., et al. (2007). Fronto-limbic and autonomic disjunctions to negative emotion distinguish schizophrenia subtypes. Psychiatry Research, 155(1), 29-44.

Williams, L. M., Liddell, B. J., Kemp, A. H., Bryant, R. A., Meares, R. A., Peduto, A. S., et al. (2006). Amygdala-prefrontal dissociation of subliminal and supraliminal fear. Human Brain Mapping, 27(8), 652-661. doi:10.1002/hbm.20208

Williams, L. M., Liddell, B. J., Rathjen, J., Brown, K. J., Gray, J., Phillips, M., et al. (2004). Mapping the time course of nonconscious and conscious perception of fear: An integration of central and peripheral measures. Human Brain Mapping, 21 (2), 64-74. doi:10.1002/hbm.10154

Winkielman, P., Zajonc, R. B., \& Schwarz, N. (1997). Subliminal affective priming resists attributional interventions. Cognition and Emotion, 11(4), 433-465. 